\title{
HISTORY OF A CASE
}

OF

\section{LIGATURE OF THE LEFT SUBCLAVIAN ARTERY,}

BETWEEN THE SCALENI MUSCLES,

ATTENDED WITH SOME PECULIAR CIRCUMSTANCES.

BY JOHN C. WARREN, M.D.,

PROFESSOR OF ANATOMY AND SURGERY IN BOSTON, U.S.A.;

HONORARY FELLOW OF THE ROYAL MEDICAL AND CHIRURGICAL SOCIETY OF LONDON, ETC. ETC.

\section{Received August 21st-Read November 25th, 1845.}

THe history of an operation for the ligature of the subclavian artery would, at this period of surgical science, seem scarcely worthy the attention of the Royal Medical and Chirurgical Society. This operation has been performed many times, and the annals of this distinguished body contain not less than twelve cases, many of them performed by the most celebrated surgeons of the age. Some peculiar occurrences, or some important practical inferences, seem necessary, to justify me in calling the Society's attention to another case. That which I have the honour to lay before them possesses peculiarities, and will, I hope, afford some practical inferences.

Some of the more remarkable circumstances in this case have occurred in former instances of the operation of ligature of the subclavian artery, while others are strictly peculiar to it. 
James Avery, on the evening of Dec. 23rd, 1843, while in a state of intoxication, slipped on the ice, fell, and struck his left shoulder against the kerbstone of the side walk. Being taken up by the bystanders, he was carried into a house, and surgical aid was called. His shoulder was found to be dislocated, and violent efforts were made to reduce the dislocation, but exactly in what manner the patient could not tell, excepting that he thought one person placed his foot, with a boot on, in the axilla. After various trials it was supposed that the dislocation was not reduced, and he was sent to the hospital for treatment.

On the next day, being examined by Dr. Hayward and myself, he presented the following appearances:- $-\mathrm{He}$ was a large, heavy, short-necked, unhealthy-looking man. His left arm was much swollen; the left shoulder enlarged and ecchymosed, so that there was no distinction of parts, and, of course, it could not be determined whether his arm was dislocated or not. The motions of the arm were accompanied with great pain. The man's age was about thirty. In this condition of the parts it was necessary, in the first place, to accomplish the reduction of the swelling, and leeches with cold applications were employed for this object.

On the next day the swelling was so much reduced, as to enable us to decide that no dislocation existed.

During the night of the third day following, Dec. 28th, he was seized with a violent fit of coughing, in which he felt something give way in his shoulder, and presumed it was thrown out of place. The house-surgeon, Mr. Townsend, being called up to him, made an extension on the arm, by which it was, as he thought, restored to its situation. On the following morning we found his shoulder and arm very much discoloured and enlarged, the arm painful, and the patient much prostrated.

On Dec. 30th, it was discovered that the man had no pulse in his left wrist, and, by examination, none could be detected in any part of the arm. This loss of pulse must 
have occurred on the night of the 28th, for before that period it had existed as usual. He now, for the first time, made it known that he had neither feeling nor motion in the arm. The loss of motion was entire, although he could, for the first five days after the accident, raise his hand to his head; there was no sensation below the scapulo-humeral articulation.

The swelling with the discolouration increased for the two or three following days, until it became enormous, the arm turning black in the axilla and on the inside of the biceps muscle. A vesication was noticed on the back of the forearm. The pulse in the sound arm was about 100. There was a pulsation above the clavicle of the affected arm, in the usual situation of the subclavian artery.

He went on quietly for some days, wholly confined to his bed by the weight and absolute immobility of his arm, when, on January 27th, 1844, a swelling was found to be forming in the axilla of the injured limb. This continued to advance with the appearance of a common abscess, excepting that the skin covering it was of a very dark colour. In seven days it pointed, and seemed ready to discharge, but did not till eight days after. On Sunday, Feb. 4th, it opened, and a gush of coagulum, with some fluid, dark-coloured blood was discharged, to the amount, it was thought, of about a pint.

Three days subsequently, namely, on Wednesday, Feb. 7th, at six o'clock in the morning, the patient being in bed, and not making any particular effort at the time, a sudden gush took place from the wound, by which the bed was at once inundated, the mattresses soaked, and blood was poured upon the floor at different points. Exhausted, and almost lifeless, he sunk into a state of syncope, and the hæmorrhage ceased. When we examined the man, a few hours after, he appeared very low ; the pulse on the sound side was exceedingly feeble, not to be discovered in the injured arm, doubtful above the clavicle.

As he was at that time too low to undergo any operation, it was agreed that, if he lived to the next day, the subclavian 
artery should, if possible, be tied. The great tumour of the shoulder above, and that of the axilla and arm below, by which the clavicle was forced upwards (for, notwithstanding the discharge of coagulum, the tumour in the axilla remained very large), rendered it doubtful whether it would be possible to get at this vessel above the clavicle, and below this bone the parts were too much deranged to admit of any operation.

By the next morning the patient had much revived; the pulse was more distinct, and a pulsation could be felt above the clavicle, in the place of the subclavian artery, as before stated. He lost a gill of blood in the morning, but not per saltum. At ten o'clock he took eighty drops of tincture of opium ; at eleven, he was carried into the operating theatre, and an operation performed as follows, on Feb. 8th, 1844.

Drs. Hayward, Townsend, Lewis, Holmes, and Parkman, attended, and assisted in the operation. A great difficulty presented itself in the outset. In consequence of the swelling of the shoulder, the tumour in the axilla, and the natural shortness of the patient's neck, the space between the shoulder and the lower jaw was almost obliterated, the shoulder rising up so high that a line passed transversely from the acromion process across the neck, when he was lying flat on his back, with his head resting upon the table, crossed the face above the lower jaw. The patient therefore had a pillow placed under his shoulders, so that his head might be depressed as much as possible. His face was turned a little to the right side. Before making any incision, search was made for the external jugular vein, but it was too much contracted to admit of being discovered through the skin.

Placing my finger on the posterior edge of the sterno-mastoid muscle, three-fourths of an inch above the clavicle, I made a transverse incision about three inches long, of which the anterior extremity corresponded with the posterior edge of the sternal head of the sterno-mastoid, and the posterior extremity with the anterior edge of the trapezius; making the point where my finger was fixed the middle of the wound. The 
fascia and platysma myoides were divided, and the sternomastoid exposed. The external jugular vein being uncovered, a branch running into it was divided, and tied; a temporary ligature was then passed around the vein, so as to draw it towards the outer part of the wound. In making these incisions, the blood, owing to its fluidity, flowed freely at every touch of the knife.

Perceiving the difficulties I should have to encounter, I at once divided the clavicular head of the sterno-mastoid muscle, and then proceeded with the handle of the knife to remove - the adipose substance immediately above and behind the clavicle. The muscles were so pallid, that it was difficult to distinguish them from the surrounding textures; the omohyoid muscle could not be discriminated from the chain of lymphatic glands lying near to it.

Passing my finger down to search for the pulsation of the subclavian artery, I perceived, corresponding with the edge of the clavicle, a strong pulsation arising from an artery of large size. This being nearly in the situation in which the subclavian would be looked for, led me to hope for a moment that I felt the latter vessel. But this delusion lasted for a moment only, for I was immediately convinced by the rolling of this artery under the finger, that it was not the fixed, immoveable subclavian, but the supra-scapular artery. It was necessary to pursue the operation with great caution, lest this artery should be divided, as it would have been difficult to have secured it, and its loss would have deprived the arm of a most important source of supply.

After pursuing the dissection with the handle of the knife and the blunt director, I passed the finger down in order to discover the tubercle of the first rib, but neither tubercle nor rib could be felt at this or any part of the operation, and thus the most exact guide to the situation of the artery and the basis on which its pulsations could be discovered, failed to appear. With the end of the finger $I$ cleared away the cellular texture, and at length discovered a faint pulsation, at the same time perceiving a cord, which $I$ ascertained to be a 
nerve; this was pushed aside, and then the pulsation being more distinct, I took this second cord, on which the finger rested, to be the subclavian artery. The aneurism-needle was then passed under the apparent artery, a ligature carried round it, and on drawing up this ligature, so far as I could judge the pulsation seemed to be suspended. On a repeated examination, however, the gentlemen around, as well as myself, were satisfied that the artery was not included in the ligature. This second nerve, which was the first dorsal, was to be removed from the surface of the artery, in order to expose the latter, but the wound was too deep, too narrow, and of consequence too dark to permit the artery to be visible. The anterior scalenus was partially visible, and passing the forefinger of the left hand to the edge of this, a good portion of the muscle was divided by the probe-pointed bistoury, introduced upon the finger. The subclavian artery then became quite sensible to the touch, and slightly distinguishable by the eye. A long aneurism-needle, with rather a short curve, was passed under the artery, and some little effort was required in turning the needle in this deep narrow wound, so as to bring the point to the opposite side of the artery. This was, however, soon accomplished, the ligature seized, and drawn out.

At this moment a slight whistling noise was heard, which I did not think of any consequence at first, having the impression that the movements of the needle had been so conducted, that the pleura could not possibly have been wounded. Being satisfied, afterwards, that some air had entered the thorax, I secured the ligature with a serre-noud, in such a way that the artery should not be elevated from the surface of the wounded pleura and fascia covering it. Then bringing together the sides of the wound with the fingers, no more air entered, and no inconvenience was experienced from that which had been previously admitted.

At 5 P.M. I visited the patient, and found he had much uncasiness in the chest from the situation of his head, which was too low. I raised his head directly, and all uneasiness left 
him, nor did he afterwards have a recurrence of pain in the chest, or complain of any other suffering. He was no sooner raised, and had expressed himself much relieved, than a loud knocking was henrd, like a person striking his knuckles upon a table. This noise was perceived all over the ward of the hospital ; I looked around to ascertain whence it proceeded, but presently suspected it came from the patient. I then placed my hand upon his chest, and found the heart knocking with great violence against the ribs. The phenomenon, which lasted two or three minutes, surprised me, the patient, and the bystanders, and I was at first at a loss to explain it; but on consideration, I think that it arose from a sudden reaction of the heart in an anæmic state, owing partly to the moving of the patient, and partly to a change in the capacity of the thorax, by which more room was given to the heart, and a pressure to which it had been for some hours subjected, was suddenly removed.

For some days the patient remained comfortable, without any particular alteration in his symptoms. His strength continued to improve steadily under a good diet, with the free use of tonics and stimulants; a large quantity of cordials being required by him, in consequence of the great loss of blood, and his previous habits of taking stimulants to excess. On the third day from the operation, three sutures, required in dressing on account of the gaping of the wound from the great enlargement of the parts, were removed, and the wound was found to have principally united. The swelling in the axilla discharged a quantity of bloody serum, but no pure blood. The arm, which had at first appeared to be in a state of partial gangrene, assumed a more healthy aspect, and the swelling gradually subsided.

On February 22nd, the thirteenth day from the operation, the ligature, being seized with the forceps, was found to lie loosely in the wound, and was removed. The pulse now ranged from 72 to 76 . Although permitted to be raised in bed occasionally, for the purpose of changing lis clothes, he 
was not allowed any active motion, until more complete closure of the wound from which the ligature had been discharged. The returning sensation had extended from the shoulder to the elbow, and even into the fore-arm.

On the afternoon of February 29th, while engaged in quiet conversation, a stream of blood, about the diameter of a common quill, was seen to issue from the unclosed part of the wound. The house-surgeon being immediately called, applied a sponge, and sent for me.

I saw him in fifteen or twenty minutes after the occurrence: he was not then bleeding, and appeared not to be much exhausted by the loss of blood. The quantity lost was supposed by the attendants to have been about a pint. In the opinion of the house-surgeon, the blood did not issue per saltum, and was of a venous colour. When it is considered, however, that it issued from a cavity two or three inches in depth, and that the aperture of the artery being small, and perhaps not corresponding exactly with the aperture of the wound, the non-appearance of an arterial jet might be accounted for, although the artery were really open. I examined the wound, placed an additional piece of sponge within it, and, to support this, another sponge on the outside, two inches in thickness, which was retained in its situation by long adhesive straps. The patient was ordered to be kept perfectly quiet, to preserve an entire abstinence from solid food, and to use a liquid regimen sparingly for two or three days.

The pieces of sponge were allowed to remain about a week, and their removal, which was accomplished without force, was not attended with bleeding or pain. From the aneurismal cavity in the axilla, a slight hæmorrhage took place a day or two subsequently.

No further alterations occurred in his symptoms until the 11 th of March, from which period until the 22nd he laboured under an attack of pulmonic inflammation, chiefly confined to the lower lobe of the left lung, accompanied with pain and soreness in the anterior part of the chest, shortness 
of breath, cough, and some black expectoration. Notwithstanding this disease, finding that his strength was failing, I considered it necessary, taking into view his former habit of using spirituous liquors, to resume the administration of cordial drinks. He was also directed to be removed from his bed to another, and occasionally to sit up, for the purpose of producing a more uniform circulation, and thus preventing those congestions which are common to patients in his situation. The tumour in the axilla, which had for some time been increasing in size, now presented a darker aspect, became very painful, and emitted a highly offensive discharge. On March 21st it threw off a round, dark-coloured lump of old coagulum, about the size of a hen's egg, to the great relief of the patient. The discharge from the wound above the clavicle, which at this time was slight, afterwards increased so as to threaten to prove troublesome. At this period he displayed a great want of mental power, arising from the extreme debility produced by the discharge. About the lst of May he had a second attack of congestive pneumonia, like the preceding, confined to the left side, from which he recovered in a few days.

In order to aid the restoration of the arm, for some weeks electro-magnetical shocks were passed through it with much benefit. By the 1st of October the tumefaction had disappeared from the arm, and motion had returned in the shoulder-joint, but not in the elbow or wrist; pulsation had not returned in any of the arteries of the arm ; sensation had gradually crept downwards from the shoulder to the extremities of the fingers, although still imperfect in the latter. A portion of the internal condyle of the os humeri having become inflamed from pressure, was necrosed; a part of the bone separated, and the wound slowly healed. The large excavation in the axilla was reduced to a fistulous tube three inches long. The wound of the operation had contracted to the depth of an inch, and the discharge from it was very slight.

In nearly this condition he remained till the early part of voL. XXIX. 
February 1845, his situation steadily, but slowly, ameliorating ; the motion, strength, and sensation of the arm constantly improving, so that an electro-magnetical current passed through the affected arm was distinctly felt in the extremities of his fingers. On February 4th, 361 days after the operation, I was able for the first time to detect a distinct pulsation in the radial artery, and subsequently one of an indistinct character in the ulnar and brachial. By the lst of April he could flex the fore-arm upon the arm, having recovered in a great degree the motions of the elbow-joint.

The patient now, June 15th, walks about freely both in and out of the house. There is still a fistulous opening in the axilla, and a slight one in the neck, extending an inch upwards from the clavicle, the cavity towards the artery being entirely healed. The sensation and motion are slowly improving. His appetite is generally good.

\section{Remarks.}

The cause of the rupture of the subclavian artery in this case is involved in some obscurity. The probability seems to be, that great violence was employed in the reduction of the dislocated humerus, and that the artery and nerves were contused by strong pressure of the operator's boot, combined with the forcible extension of the arm. The vessel did not rupture immediately, because its coats were contused and not torn asunder, but a separation of the contused parts took place in consequence of the violent efforts of coughing on the fifth day after the accident. This is the best hypothesis we can form on the subject, though not perfectly satisfactory, for the arm was so much swollen at the time the patient was first examined, that it was impossible to distinguish the effects of the accident from the effects of the force used to remedy it. 
The loss of sensation at the time of the rupture of the artery may be attributed to the compression of the nerves from the prodigious effusion of blood into the cellular texture of the axilla and arm. The return of both sensation and motion must be attributed to the slow restoration of the nervous current after the compression was removed.

The next circumstance that presents itself in the course of the history is the formation of an apparent abscess in the axilla. The swelling occurred in a very gradual way, slowly increasing for at least twenty days, and assuming a pointed summit, which softened by degrees, and, for more than a week, threatened to open daily, seeming to invite the knife so strongly, that more than one intelligent surgeon was shocked at the apparent neglect of an incision. This had not, however, the aspect of a common abscess, nor had it exactly that of a sanguineous tumour, so that there was nothing to cause great alarm as to the primary effect of its opening. It was thought best, however, to leave it to be opened by the hand of Nature in a case so complicated, and having so many unfavourable circumstances.

The interval between the opening of the abscess and the occurrence of the great hæmorrhage - three days-might at first excite surprise, but this will cease when it is recollected that the cavity of the abscess and that of the aneurism were obviously distinct from each other; that the opening of the former would not necessarily involve that of the latter; and, in fact, we entertained strong hopes that the lacerated artery would be, or, to speak with more precision, actually was, plugged by an internal coagulum at the time the abscess opened. This might possibly have been the fact, and the cough, which still continued, might have forced open the lacerated artery, and thrown out the internal plug. The hæmorrhage which occurred from the aneurism was as great as the patient could support without expiring, and, through the day on which it happened, it was doubtful whether he could survive twelve hours. The operation for tying the artery was done as early as the circumstances of the patient would justify. 
It was done on the emergency of the occasion, and, of course, without that preparation which surgeons desire when they are called on to do unfrequent and dangerous operations, although, in a lapse of years, I had had occasion to put a ligature on the subclavian artery in the dead subject more than a hundred times.

The admission of air into the pleura during the operation has happened to other surgeons. In such a case as this it would be difficult to take any course which would certainly avoid it; but $I$ have adverted to it distinctly and repeatedly, that others, apprised of the possibility of its occurrence, may be better prepared to regulate their movements in such way as to avoid it.

Immediately after the operation, there was no circumstance worthy of remark, excepting that the arm, which was covered with cotton in the usual way, retained its natural temperature without any extraordinary precautions. This is the more remarkable, because the patient had lost a large quantity of blood the day before, and the function of the nerves of the limb was interrupted.

The occurrence of secondary hæmorrhage, twenty days after the operation, did not surprise me; for, although the patient appeared to be doing well at that time, yet the arm was in so dangerous a state from the paralysis, swelling, ecchymosis, threat of gangrene at various points, but, more than all, from the proximity of collateral arteries to the ligature, that I was led to place little confidence in the hope of this man's reocvery. 